\title{
The COVID-19 Pandemic: A View from New York City
}

\author{
Domenick J. Masiello ${ }^{1,2}$ \\ ${ }^{1}$ Department of Family \& Community Medicine, New York Medical \\ College, Valhalla, New York, United States \\ 2 Department of Osteopathic Manipulative Medicine, Touro College of \\ Osteopathic Medicine, Middletown, New York, United States
}

\begin{abstract}
Address for correspondence Domenick J. Masiello, DO, DHt, C-SPOMM, 141 East 55th Street, New York, NY 10022, United States (e-mail: dr.djmasiello@gmail.com).
\end{abstract}

Homeopathy 2020;109:163-166.
Abstract
Keywords
- Gelsemium
- Senega
- Antimonium ars.
- Psorinum
- pandemic
- epidemic
- COVID-19
- SARS-CoV-2
- reverse transcriptase polymerase chain reaction assay
- Genus Epidemicus
- coronavirus

This article provides a view of homeopathic clinical practice in the New York City area in the first few months of 2020 as the coronavirus disease 2019 (COVID-19) pandemic began to evolve in the United States. Key symptoms used to generate a short list of potentially curative remedies are given, and the pandemic syndrome is viewed as appearing in stages or as having various clinical manifestations each with its own main remedy. The severe acute respiratory syndrome coronavirus 2 (SARS-CoV-2) is briefly described, as are the preliminary presenting signs and symptoms of COVID-19 infection. Several clinical examples are given, some with positive laboratory confirmation.

\section{Introduction}

The history of the homeopathic management of epidemic diseases dates back to the battle of Leipzig in 1813 when Samuel Hahnemann treated an outbreak of typhus. He treated 180 patients (losing only two) and published his results the following year in Allegemeine Anzeiger der Deutschen. ${ }^{1}$ Hahnemann's guidance for treating an epidemic disease appears in the Organon (par. 100-102). ${ }^{2}$ He advised the following: (a) assume every epidemic to be new and unknown; (b) examine it thoroughly in all its details; (c) the totality of the signs and symptoms is revealed only after several cases are observed, never from any one patient; (d) the ordinary symptoms and the more unusual symptoms constitute the characteristic picture of the disease; (e) all patients who catch the epidemic are suffering from the same disease; (f) in subsequent cases the appropriateness of the remedy will be corroborated or a more appropriate one will be revealed. In 1900, Kent echoed

received

May 6, 2020

accepted after revision

May 20, 2020

published online

July 14,2020

Hahnemann's instructions in the third chapter of his Lectures on Homeopathic Philosophy ${ }^{3}$ but noted that there might be a group of a six to seven possible epidemic remedies which must be studied carefully before a prescription can be made.

Every winter presents an opportunity to apply the principles of homeopathy to the treatment of epidemics. Starting in late December 2019, a flu-like illness made its appearance in the New York City area. It was characterized by a lowgrade fever, dull headache, body aches (muscles, not joints or bones), and mild pharyngitis with sub-mandibular adenopathy. Mental dullness and fatigue seemed to be the symptoms that were outstanding for most patients. The other feature was duration-the symptoms seemed to last for 2 or 3 weeks despite improvement with Gelsemium. The behavior of the illness itself resembled Gelsemium: slowness, apathy, listlessness, languor and torpor-it just seemed to drag on and on. Relapses were common and changes in the potency of Gelsemium were required.

Copyright $\odot 2020$ The Faculty of Homeopathy
DOI https://doi.org/ 10.1055/s-0040-1714052. ISSN $1475-4916$. 
As is frequently the case with influenza, some patients make a partial recovery and are troubled by a persistent symptom or two. The most common post-Gelsemium symptom this year was a chronic cough. Next in frequency was a sore throat and profuse nasal discharge, sometimes thick and sometimes accompanied by paroxysmal sneezing. One patient presented with blepharitis as her chief complaint. Over the years in these viral post-treatment scenarios, I have often availed myself of an invaluable small rubric from Boericke's Materia Medica with Repertory. ${ }^{4}$ It can be found under Respiratory System: Cough: Cause: Influenza. The rubric contains the following remedies: Allium cepa, Eriodictyon, Hyoscyamus, Kali bichromicum (Kali-bi), Kali sulphuricum (Kali Sulph), Kreosotum, Pix Liq., Sanguinaria, Senega, Stannum, and Strychninum. Statistically, the most frequently used remedies from this rubric by far have been Kali-bi, Sanguinaria and Senega.

\section{Evolution of a Genus Epidemicus}

By early February 2020, it became clear that this illness was not influenza but perhaps early cases of the novel coronavirus. After seeing several post-Gelsemium cases and finding that many of their symptoms were covered by Senega, that remedy was added to the list of those needed. By late February, a pattern emerged which indicated that this epidemic might have at least three phases or facets to it: the first phase is represented by Gelsemium.

In the second phase, Senega was needed when some or all of the following symptoms made their appearance: cough, shortness of breath, persistent headache, chest pain, chest heaviness, sore throat, difficult expectoration, rattling mucus, and persistent fever. Several days of b.i.d. dosing of Senega were required to make the turn back to health. The last symptom to resolve is the patient's energy level which can take another week. Senega is especially needed for respiratory conditions in the elderly. I have not yet seen the more unusual Senega symptoms such as the feeling that the chest is too narrow or coughing that ends in a sneeze.

During this second phase a few other remedies have been needed to complete the treatment: Eupatorium perfoliatum (persistent headaches, worse from moving the eyes), Phosphorus for a vague 'spacey' dizziness, Sulfur (which follows Senega well) to get back to a sense of normal and clean up minor symptoms, and Kali-bi for residual headache at the center of the forehead or bridge of the nose, for throat and nasal dryness with anosmia and ageusia. Senega does have distortion of the sense of taste but it typically will not completely treat the anosmia.

Kent's chapter on Senega ${ }^{5}$ is most informative. Although only partially proved, Senega is clearly a lung remedy with an affinity for the mucus membranes of the air passages. Kent tells us that it is deeper than Bryonia, and that it is often overlooked in favor of Bryonia. The chest rattling resembles Antimonium tartaricum and the stringy, tenacious mucus resembles Kali-bi. Other remedies in Kent's differential include Phosphorus, Arsenicum album, Lachesis, Spongia, and Rumex.
Many of the symptoms of coronavirus disease 2019 (COVID-19) patients can be found in the Senega chapter of Kent's Lectures on Homeopathic Materia Medica ${ }^{5}$ and in the Senega chapter of Clarke's Dictionary of Practical Materia Medica. ${ }^{6}$ These include headache (forehead and orbits), swelling of the eyelids, photophobia, frequent and violent sneezing, dryness of the nasal passages, dryness of the mouth with a metallic taste, dry, sore and burning throat, copious and tenacious mucus in the throat, rattling of mucus in the chest which is difficult to expectorate, chest pains worse at rest, asthmatic troubles made worse by ascending the stairs, incessant cough made worse by motion, shortness of breath while climbing the stairs, nausea, vomiting, diarrhea, and great weakness with physical and emotional depression.

The third phase of this disease may be represented by the remedy Antimonium ars. This is my least defined remedy for this condition because it is based on only two clinical cases, which were managed remotely. One case was that of a 77-year-old female, with a past history of chronic conjunctivitis, chronic blepharitis, seasonal and environmental allergies, pruritus senilis, lumbago and chronic insomnia, who had done quite well under my homeopathic and osteopathic care. She initially responded well to Gelsemium but required a dose of Natrum sal. to restore her mood and energy level. However, 2 weeks later she again relapsed and she felt that she had yet another bad cold with much sneezing, sinus congestion, egg-white nasal discharge and fatigue. Senega was prescribed which helped the symptoms for 2 to 3 days but a week later she called with the following symptoms: a dull, heavy frontal headache above the eyes, fever ranging from 99.2 to 100.5, itching in the throat and trachea with a persistent cough. She felt like she was 'drowning in her secretions' but she could not expectorate. She was extremely cold and could not get warm despite being under the covers with a hot-water bottle. The symptoms were repertorized using RadarOpus software, with the analysis set to 'small remedies'. Because of the intensity with which the patient described her chilliness, Antimonium ars. 200c was selected and mailed to the patient, to be taken mixed in water b.i.d. Two days after taking the remedy, the fever broke, the cough improved, and her energy level returned to normal. She had 2 days of frequent, soft, paste-like stool which was most likely viral shedding. It then seemed that when Senega fails to bring about a cure and the patient is still febrile with extreme chilliness, has signs of bronchitis or an incipient pneumonia, Antimonium ars. can rally the patient.

Antimonium ars. is a rare or small remedy with little more than a paragraph of description in standard homeopathic references. Volume I, No. 1 of the Homeopathic Recorder, published in January 1886 (pp. 10 to 12), contains a brief mention of Dr. Mattes who summarized four cases of Stibium (Antimonium) Arsenicosum which he quotes from Allg. Hom. Zeit, Nos. 2, 5, and 8 from 1885 . Reading these cases, it is easy to see the amount of lung and other pathology that was cured in children and the elderly with this remedy. Pneumonias with exudation, asphyxia, and pericarditis with effusion 
were cured. Based on the description of the signs and symptoms, patients were cyanotic, febrile, diaphoretic, tachypneic, with consolidation involving most of the lung fields. These were cases of post-measles and post-influenza catarrhal pneumonias rapidly cured with low potency Antimonium ars. Although highly speculative at this point, this small remedy may be useful for hospitalized patients or home-bound patients in danger of imminent hospitalization.

\section{COVID-19 Confirmation}

Up to this point, patients with a flu-like disorder were responding well to homeopathy; however, without laboratory confirmation it was impossible to know if they were suffering from COVID-19 infection. Confirmation was received by mid-March when prescribing for a new patient with a complex medical history. The patient was a 35-yearold man with a 17-year history of allergies, asthma and eczema, previously treated unsuccessfully with a variety of homeopathic remedies prescribed by several homeopaths. His current medications were the anti-histamine levocetirizine, the anti-depressant fluoxetine, and the biologic drug dupilumab. The patient was told to take Psorinum 30c mixed in water on March 17 and was instructed to call the office on March 24. He was told not to change any of his current orthodox medication. He was also treated with osteopathic manipulation to the cranium and cervical, thoracic and lumbosacral areas.

On March 24, 2020, the patient called the office and reported that after the Psorinum 30c his sleep was better and he felt that his skin seemed more 'stable'. He also reported that at the beginning of March 22 he began to experience flu-like symptoms: low-grade fever, aching muscles and fatigue. He was instructed to take the highest potency of Gelsemium that he could obtain and to repeat it once a day. He managed to get Gelsemium 30c and took a dose the same day (March 24): he experienced a spike in his temperature and profuse sweats later that night.

On March 29, he called again to report that despite several more doses of Gelsemium 30c the tiredness and aching muscles continued and on March 26 chest and nasal congestion had started. This was accompanied by a hacking cough which was worse at night. By March 28 he began to wheeze and still had the cough and fatigue. On March 29, his breathing became more difficult with shortness of breath, dry hacking cough and wheezing, but he was afebrile. He also began to lose the sense of taste and smell. He was advised to take a dose of Senega 200c and to repeat it once a day by mixing it in water and shaking or stirring the solution.

Any possible confusion about the significance of the symptoms that appeared after Psorinum 30c was clarified when he called again on April 7 and mentioned that he had tested positive for the COVID-19 virus on March 30, one day after having taken Senega 200c. A reverse transcriptase polymerase chain reaction (RT-PCR) assay on a swab of his nasopharyngeal secretions was performed by Bio Reference Labs. He also said that after the first dose of Senega 200c on March 29, the wheezing and shortness of breath improved within a few hours. From March 31 to April 6, his symptoms were mainly a mild dry cough, a feeling of chest heaviness/ tightness, mild wheezing and fatigue. On April 7, he began to feel like 'his normal self' again. Approximately 4 weeks later, IgG antibodies to the COVID-19 virus were detected.

Subsequently, two more patients treated successfully with Senega have tested positive for IgG antibodies to COVID-19. In addition, a 2-year-old child, who was given a dose of Senega 200c every 2 weeks as a preventative and who was asymptomatic, also tested positive for IgG antibodies. Eleven weeks after the 77-year-old patient recovered from her illness using Arsenicum-ars., she too tested positive for IgG antibodies to COVID-19.

\section{Discussion}

The severe acute respiratory syndrome coronavirus 2 (SARS$\mathrm{CoV}-2$ ) has been identified as the cause of the coronavirus pandemic of 2020. First cases were identified in Wuhan, China, in December 2019. This global syndrome has since spread exponentially and the World Health Organization declared the disease a pandemic in March 2020.

The coronaviruses (Coronaviridae) were first discovered in the1960s. They are a family of single-stranded RNA viruses, some of which are pathogenic. The alpha-coronavirus and the beta-coronavirus strains are thought to have originated from bats (Rousettus leschenaultii). ${ }^{7}$ The clinical sequelae are wide-ranging, from mild cold symptoms to respiratory failure and death. MERS-CoV (Middle East respiratory syndrome coronavirus), SARS-CoV and SARS CoV-2 are beta-coronavirus strains and are known to cause respiratory symptoms, respiratory distress and death. ${ }^{7}$

Direct transmission of the virus is via respiratory droplets spread by coughing and sneezing and via contact with fomites in the immediate environment of infected persons. ${ }^{8}$ The SARS-CoV viruses enter the cells via an endocytosis pathway using a spike protein to bind to the angiotensinconverting enzyme 2 receptor. ${ }^{9}$

Symptomatic patients who test positive (via RT-PCR assay) may display a wide range of signs and symptoms, including fever (99\%), fatigue (70\%), dry cough (60\%), lymphopenia (70\%), prolonged prothrombin time (58\%), elevated lactate dehydrogenase (40\%), and chest computerized tomography scans showing either patchy shadows or ground glass opacities in the lung fields. ${ }^{10}$ The severity of symptoms seems to vary, affecting a disproportionally high number of older patients with pre-existing medical conditions.

There are no known definitive conventional therapies for the disease and there are currently no vaccines available. Conventional management is supportive care alone but there are several studies underway that aim to identify safe and effective orthodox drug therapies. Without definitive conventional medicines available, homeopathy provides an alternative treatment for those COVID-19 positive patients with mild to moderate symptoms who are recovering at home, potentially sparing them the need for hospitalization. Such treatment is consistent with the historical use of homeopathic interventions during epidemics. 


\section{Conclusion}

There are various lists of COVID-19 homeopathic remedies making the rounds on the internet these days. Some lists have more than 20 remedies on them and it is unlikely that they can all be the Genus Epidemicus. Other contributors have declared that they have discovered one single simillimum for this pandemic. Still others have declared that a remedy cannot be used prophylactically during a pandemic, seeming to ignore Hahnemann's scarlet fever experience, Boericke's list of prophylactics in the Generalities section of his repertory, and Clarke's experience with prevention and nosode use during influenza epidemics, as noted in the Influenza section of The Prescriber. ${ }^{11}$

It is important to realize that there are no physicians alive today, homeopathic or otherwise, with any real-world experience of treating a pandemic like this one. Therefore, there are no COVID-19 pandemic experts, no 'modern masters' as it were; we are all learning as we go. The Spanish flu pandemic of 1918 lasted about 2 years and attacked in three waves before it departed. However, before it did, homeopaths were able to apply the principles of homeopathy and save many lives. We can learn from an article entitled, Homeopathy in Influenza-A Chorus of Fifty in Harmony, by W.A. Dewey, MD, from the University of Michigan. It appeared in The Journal of the American Institute of Homeopathy, in May 1921. In the article we can get a glimpse of the distribution and devastation of the pandemic, the lower mortality rate among homeopathy patients, and the chief remedies used for treatment.

We are at the very beginning of this pandemic and there will no doubt be changes in the expression of this virus and much to learn about individual susceptibility to it. Hopefully, we can develop a short list, a constellation of several 'stars' to combat this miasm. My observations on Gelsemium, Senega and Antimonium ars. are based on my initial experience treating this pandemic in New York City, one of the global epicenters of the disease. Most of the approximately 30 cases treated were in person and some by phone or video session. As antibody testing becomes more available, it is incumbent upon all of us to test our cured patients and document the efficacy of our treatments. If this pandemic is anything like the Spanish Flu, we may be treating the after-effects of this disease for years to come. Twenty years after the end of the Spanish Flu pandemic, an article by Guy Beckley Stearns, MD, entitled, Influenzins, was published in The Homeopathic
Recorder (Vol. LIII, No. 10, November 1938). In it, he documented some chronic manifestations of what could be called the influenza miasm. Stearns argued that influenza had altered the expression of other infectious diseases and documented the use of influenza nosodes in the treatment of difficult chronic cases. Only time will tell if there will be a chronic COVID-19 miasm and a viable COVID-19 nosode.

\section{Conflict of Interest}

None declared.

\section{Acknowledgement}

My thanks to all homeopathic patients worldwide for their continued confidence in and support of this healing method and to their homeopathic physicians who are now assembling various databases of cured COVID-19 cases for future research and archival purposes. I want to especially thank each of my patients mentioned in this article for providing their informed consent for relevant case details and testing results to be used anonymously for academic purposes.

\section{References}

1 Cook T. Samuel Hahnemann - His Life and Times. Middlesex: Homeopathic Studies Limited; 1993:104

2 Hahnemann S. Organon of Medicine. 6th ed. Los Angeles, CA: J.P. Tarcher; 1982

3 Kent, J. Lectures on Homeopathic Philosophy, Berkeley: North Atlantic Books; 1979

4 Boericke W, Boericke O. Pocket Manual of Homeopathic Materia Medica with Addition of a Repertory. 9th ed. Philadelphia, PA: Boericke and Runyon; 1927

5 Kent J. Lectures on Homeopathic Materia Medica. 3rd ed. Philadelphia, PA: Boericke \& Tafel; 1923

6 Clarke J. A Dictionary of Practical Materia Medica. London: The Homeopathic Publishing Company; 1900

7 Valencia DN. Brief review on COVID-19: the 2020 pandemic caused by SARS-CoV-2. Cureus 2020;12:e7386

8 World Health Organization. Modes of Transmission of Virus causing COVID 19: Implications for IPC Precaution Recommendation. Available at: https://www.who.int/news-room/commentaries/detail/modes-of-transmission-of-virus-causing-covid-19implications-for-ipc-precaution-recommendations

9 Cui J, Li F, Shi ZL. Origin and evolution of pathogenic coronaviruses. Nat Rev Microbiol 2019;17:181-192

10 Wang D, Hu B, Hu C, et al. Clinical characteristics of 138 hospitalized patients with 2019 novel coronavirus-infected pneumonia in Wuhan, China. JAMA 2020;323:1061-1069

11 Clarke J. The Prescriber. Essex: Health Sciences Press; 1972 